\title{
Efeitos de Idade e Sexo no Autoconceito de Crianças Pré-Escolares
}

\section{Age and Sex Effect on the Self-Concept of Preschool Children}

\author{
Alexandra Pinto, Ana Gatinho, Carla Fernandes \& Manuela Veríssimo* \\ ISPA - Instituto Universitário de Ciências Psicológicas, Sociais e da Vida, Lisboa, Portugal
}

\begin{abstract}
Resumo
Poucas são as investigações sobre o autoconceito realizadas com crianças de idade pré-escolar. Neste estudo procuramos contribuir para uma melhor compreensão do desenvolvimento normativo do autoconceito, no período pré-escolar, nomeadamente, através da análise dos efeitos que o sexo e a idade têm no mesmo. Tratando-se de um estudo longitudinal, a amostra foi composta por 83 crianças portuguesas, avaliadas no seu autoconceito aos quatro e aos cinco anos de idade, através da Pictorial Scale of Perceived Competence and Social Acceptance for Young Children - PSPCSA. As crianças apresentaram resultados muito elevados em todos os domínios do autoconceito, em ambas as idades Os dados parecem indicar que os elevados valores do autoconceito começam a declinar já no fim do período pré-escolar, pelo menos em alguns domínios, sugerindo, dessa forma, um desenvolvimento diferenciado do autoconceito, consoante os diferentes domínios avaliados. Finalmente, parecem existir diferenças no autoconceito relacionadas com o sexo das crianças, favorecendo os rapazes. Os nossos resultados contribuem para uma melhor compreensão do processo de desenvolvimento do autoconceito.

Palavras-chave: Autoconceito, pré-escolar, género.
\end{abstract}

\begin{abstract}
There are few investigations on the self-concept of preschool children. The aim of this study is to contribute to a better understanding of the normative development of self-concept in preschool children, particularly by analyzing the effects of gender and age. In a longitudinal study, our sample consisted of 83 Portuguese children, and data were collected at four and five years old. Self-concept was measured by the Pictorial Scale of Perceived Competence and Social Acceptance for Young Children - PSPCSA. Children presented very high results in all areas of self-concept at both ages. Data seem to indicate that self-concept starts to decline towards the end of the preschool period, at least in some areas. Results suggest a differentiated process of self-development, according to the different domains assessed. Finally, there seem to be differences in self-concept related to the children's gender, favoring boys. Our results contribute to a better understanding of the developmental process of self-concept.

Keywords: Self-concept, preschool, gender.
\end{abstract}

O estudo do autoconceito tem uma longa história em Psicologia, uma vez que as crenças e sentimentos sobre si próprio têm um papel determinante no desenvolvimento individual (Oyserman, Elmore, \& Smith, 2012). No entanto, pouca tem sido a investigação nesta área realizada com crianças de idade pré-escolar (Marsh, Ellis, \& Craven, 2002; Pinto, Gatinho, Silva, Veríssimo, \& Santos, 2013).

Há duas valências conceptuais que se destacam na investigação nesta área: (a) um conceito mais global, relacionado com uma avaliação emocional / afetiva do indivíduo sobre si mesmo, em termos gerais, descrevendo o valor que a pessoa se dá, ou seja, a extensão na qual o próprio se vê como válido, significativo e digno e que inclui termos como autoestima, autoavaliação, e autovalor; (b) um conceito mais específico, multidimensional e por vezes hierárquico, que geralmente se refere a uma descrição do self, sem necessariamente incluir uma avaliação, e que está relacionada com a cognição, incluindo termos como autocognição, autoconceito ou autoimagem (Oyserman et al., 2012).

Dois dos termos mais utilizados nas investigações sobre esta temática têm sido o autoconceito, ou seja, a perceção 
dos indivíduos quanto à sua competência em diferentes domínios, como o académico, social, comportamental e atlético (Harter, 2012; Manning, 2007; Oyserman et al., 2012) e a autoestima, relacionada com a capacidade dos indivíduos realizarem uma avaliação global, positiva de si mesmos, com forte componente afetiva (Peixoto, 2003).

Tal como em relação à terminologia, existe uma grande profusão de modelos teóricos explicativos do autoconceito: (a) unidimensionais; (b) multidimensionais; (c) taxonómicos; (d) compensatórios; (e) de referência interna / externa; (f) multidimensionais hierárquicos. Nos modelos multidimensionais, o autoconceito é concebido como uma entidade para a qual contribuem diversos fatores ou dimensões. A investigação tem demonstrado evidência de suporte aos modelos multidimensionais do autoconceito (Marsh \& Hattie, 1996). Os postulados teóricos de Harter (2012) compreendem o autoconceito segundo este tipo de modelo. Como tal, os seus instrumentos possuem diferentes dimensões. A Pictorial Scale of Perceived Competence and Social Acceptance for Young Children (PSPCSA; Harter \& Pike, 1984) engloba quatro dimensões: (a) Competência Cognitiva; (b) Competência Física; (c) Aceitação dos pares; (d) Aceitação Materna.

A PSPCSA é uma extensão modificada da escala original de Harter (1983) Perceived Competence Scale for Children. A extensão da escala original, para crianças pequenas, obrigou à sua modificação em diversos aspetos: (a) utilização de um formato pictórico, adequado ao sexo da criança; (b) criação de duas versões, uma para as idades pré-escolares ( 4 e 5 anos de idade) e outra para os primeiros anos de escolaridade (6 e 7 anos de idade); (c) omissão da subescala de autovalor geral, já que os dados empíricos e teóricos de Harter (1983) sugerem que as crianças com idade inferior aos 8 anos não conseguem determinar, de forma fiável, o seu autovalor geral; (d) definição de dois constructos principais, a competência percebida e a aceitação social percebida, cada uma com duas subescalas. Desta forma, a PSPCSA possui as quatro subescalas acima nomeadas, que se agregam em dois compósitos: aceitação social e competência percebidas. Harter e Pike (1984) encontraram evidência empírica de suporte a esta estrutura.

$\mathrm{Na}$ sua revisão de instrumentos para a investigação do autoconceito em crianças, Byrne (1996) identificou a PSPCSA como uma medida promissora, por apresentar um formato pictorial e específico para o sexo da criança e possuir itens apropriados em termos desenvolvimentais. Este tem sido um instrumento bastante utilizado, apresentando resultados satisfatórios em termos de validade. Por exemplo, a investigação em crianças de idade pré-escolar demonstrou que crianças de 4 e 5 anos são capazes de fazer julgamentos sobre domínios específicos da sua competência (e.g., Harter \& Pike, 1984; Marsh, Craven, \& Debus, 1991; Marsh et al., 2002).

\section{Efeitos da Idade no Autoconceito}

A investigação nesta área demonstrou que as crianças tendem a providenciar julgamentos demasiado positivos / elevados sobre as suas competências, traduzindo-se numa indiferenciação entre as facetas do autoconceito consideradas (e.g., Harter, 1983, 2012; Harter \& Pike, 1984). Este facto tem sido atribuído à limitada capacidade de processamento de informação das crianças pré-escolares (e.g., não conseguem realizar comparações sociais - dependem de comparações temporais -, e não conseguem distinguir autoconceitos reais de ideais), bem como aos processos de socialização desta idade, com os adultos a providenciarem feedbacks extremamente positivos aos esforços das crianças (Harter, 2012; Stipek \& Mac Iver, 1989). Desta forma, Harter (2012) afirma que as autoavaliações das crianças pequenas podem ser consideradas normativamente irrealísticas. Vários estudos suportam estas assunções, reportando tanto o incremento das correlações dos resultados do autoconceito com heteroavaliações de outros significativos, como o aumento das correlações desses resultados com indicadores de desempenho, como por exemplo entre o autoconceito académico e medidas de realização escolar (e.g., Eccles, Wigfield, Harold, \& Blumenfeld, 1993; Marsh, Craven, \& Debus, 1998). Esta tendência parece declinar nos primeiros anos de escolaridade, facto pelo qual a maioria dos estudos realizados sobre os efeitos da idade no autoconceito de crianças referem a existência de uma diminuição nas diferentes dimensões consideradas (e.g., Cole et al., 2001; Eccles et al., 1993; Marsh et al., 1991; Stipek \& Mac Iver, 1989). No entanto, alguns autores (e.g., Cole et al., 2001; Marsh et al., 1998) sugerem que poderão existir variações nos padrões de mudança ao longo do tempo, nas diferentes facetas do autoconceito, podendo algumas permanecer estáveis e outras diminuir de forma estável.

Sintetizando os resultados obtidos na investigação sobre este assunto, com crianças de idade pré-escolar, alguns estudos demonstraram não existirem diferenças estatisticamente significativas devidas à idade, no autoconceito / self (Ackerman \& Dozier, 2005; McCarthy, 1998; Verschueren, Marcoen, \& Schoefs, 1996). Ackerman e Dozier (2005) analisaram crianças de 5 anos de idade em relação às suas representações do self, utilizando a Puppet Interview. McCarthy (1998) referiu-se ao mesmo conceito, e utilizou tanto a Puppet Interview, como uma metodologia de histórias incompletas com uma família de bonecos, com crianças de 4 e 5 anos de idade. Finalmente, Verschueren et al. (1996), investigaram o modelo interno do self, através da Puppet Interview modificada, a autoestima, através de uma metodologia que analisa manifestações comportamentais de autoestima e de um questionário pictórico criado para o efeito e o autoconceito por meio da PSPCSA, com crianças de idade média de 5 anos e 3 meses.

Por outro lado, alguns estudos revelaram a existência de correlações negativas significativas entre a idade das crianças e a perceção do self, indicando que crianças mais novas têm perceções do self mais elevadas, comparativamente a crianças mais velhas (Colwell \& Lindsey, 2003; Mantzicopoulos, 2004). Colwell e Lindsey (2003) analisaram as representações do self de crianças com uma idade média de 5 anos, por meio de uma entrevista de fantoches e Mantzicopoulos (2004) analisou as autoperceções do self 
através da PSPCSA em crianças com uma idade média de 5 anos de idade.

Contudo, outros estudos encontraram resultados no sentido contrário (Clark \& Symons, 2000; Goodvin, Meyer, Thompson, \& Hayes, 2008). Clark e Symons (2000) analisaram tanto o autoconceito, com a PSPCSA, como a autoestima, através da versão modificada da Puppet Interview, em crianças com 5 anos, tendo encontrado correlações positivas significativas entre a idade das crianças e a positividade da autoestima. Por sua vez, Goodvin e colaboradores (2008) realizaram um estudo longitudinal, avaliando as crianças aos 4 e aos 5 anos de idade e analisaram o autoconceito das crianças através do Child Self-View Questionnaire, não tendo encontrado diferenças no autoconceito devidas à idade das crianças.

A investigação tem demonstrado que apesar da diminuição dos resultados nas diferentes dimensões do autoconceito, durante a infância e pré-adolescência, as avaliações globais (autoconceito global, representações do self ou autoestima), mantêm alguma estabilidade (ver Peixoto, 2003). Desta forma, não será de estranhar que se encontrem resultados diversos na investigação.

\section{Efeitos do Sexo no Autoconceito}

Poucas são as investigações que analisaram diferenças no autoconceito em função do sexo em crianças de idade pré-escolar. Historicamente, os estudos que analisaram esse tipo de diferenças focaram-se em valores globais de autoconceito ou autoestima. Ainda assim, a pouca investigação produzida sobre este assunto, em crianças mais velhas e adolescentes, com instrumentos adequados a cada idade, reportou a existência de diferenças significativas nas dimensões específicas do autoconceito, apontando para a existência de diferenças explicadas pelos estereótipos sexuais, com os rapazes a apresentarem autoconceitos mais elevados nas dimensões físicas (competência desportiva/ atlética e aparência física; e.g., Eccles et al., 1989; Harter, 1983) e de competência matemática (e.g., Eccles et al., 1993; Marsh, Smith, \& Barnes, 1985) e as raparigas a percecionarem-se como mais competentes nas dimensões académicas relacionadas com o domínio da língua materna (e.g., Eccles et al., 1993; Marsh et al., 1985), na manutenção de amizades íntimas na adolescência (e.g., Harter, 1988) e como melhor comportadas (e.g., Harter, 1985, 2012).

Alguns investigadores têm sugerido que as diferenças em função do sexo estão presentes logo no início da escolarização (e.g., Eccles et al., 1993; Marsh et al., 1991), embora a investigação em crianças pré-escolares e no início do período escolar não tenha encontrado, de forma consistente, diferenças nas autoperceções das diferentes dimensões do autoconceito (e.g., Harter \& Pike, 1984; Mantzicopoulos, 2004). Ainda assim, Marsh e colaboradores $(1991,1998)$, com crianças dos 5 aos 8 anos de idade (1991) e de 6 e 7 anos (1998), encontraram efeitos do sexo, favorecendo os rapazes, nas autoperceções de competência física, notando que essas diferenças tendiam a aumentar com a idade. A investigação realizada sobre este assunto, no período pré-escolar, tem avaliado sobretudo diferenças a níveis mais globais do autoconceito ou do self e autoestima. Os resultados têm sido semelhantes, não se encontrando, na sua maioria, diferenças significativas (Ackerman \& Dozier, 2005; Bird \& Reese, 2006; Emídio, Santos, Maia, Monteiro, \& Veríssimo, 2008; Goodvin et al., 2008; Mantzicopoulos, 2004; McCarthy, 1998; Verschueren et al., 1996). No entanto, alguns estudos encontraram diferenças de sexo, favorecendo as raparigas (Clark \& Symons, 2000; Gullón-Rivera, 2013). Finalmente, Marsh e colaboradores (2002) reportaram tendências significativas para as raparigas terem resultados mais elevados no autoconceito relacionado com os pares (social) e os rapazes no autoconceito físico.

O presente trabalho pretende contribuir para as lacunas apontadas: (a) contribuir para uma melhor compreensão do desenvolvimento normativo do autoconceito; (b) investigar se no fim do período pré-escolar já se começa a observar o referido declínio nas autoavaliações do autoconceito; (c) investigar se existem diferenças devidas ao sexo, no autoconceito das crianças pré-escolares; (d) investigar se no período pré-escolar já se encontram diferenças de idade e sexo nas diferentes dimensões do autoconceito.

\section{Método}

\section{Participantes}

Selecionaram-se para este estudo 83 crianças, de um total de 340 , a quem foi administrada a PSPCSA para um estudo longitudinal mais vasto, previamente aprovado por comissão de ética. Nesta seleção presidiu o critério de possuir respostas das crianças em dois pontos temporais, aos 4 e aos 5 anos de idade. A falta de dados, numa ou noutra idade, que obrigou à redução de amostra, correspondeu a faltas das crianças nos dias das recolhas de dados, mudanças e/ou entradas de escola e outros fatores de carácter normativo num estudo deste tipo. As crianças pertenciam a dois estabelecimentos do ensino pré-escolar da região de Lisboa, um deles privado e o outro, uma Instituição Particular de Solidariedade Social (IPSS). As crianças foram recrutadas junto dos pais, para participarem num estudo longitudinal mais vasto, em cada uma das salas de aula pré-escolar de cada estabelecimento de ensino. Os pais receberam informações sobre o projeto de investigação e seu objetivo, e preencheram um consentimento informado. A PSPCSA foi administrada às crianças quando elas tinham 4 anos (meses $M=54,95 ; D P=4,06)$ e 5 anos de idade (meses $M=69,40 ; D P=7,41$ ). Relativamente às famílias das crianças, o nível de escolaridade materna varia entre os 9 e os 23 anos de escolaridade $(M=15,00 ; D P$ $=3,04)$ e as habilitações paternas entre os 4 e os 19 anos $(M=15,1 ; D P=3,33)$. As mães tinham em média 35,8 anos $(D P=4,67)$ de idade e os pais 38,3 anos $(D P=6,6)$.

\section{Instrumento}

Pictorial Scale of Perceived Competence and Social Acceptance for Young Children (PSPCSA; Harter \& Pike, 1984; Mata, Monteiro, \& Peixoto, 2008). Instrumento pictórico que parte do pressuposto que as crianças pré- 
Pinto, A., Gatinho, A., Fernandes, C. \& Veríssimo, M. (2015). Efeitos de Idade e Sexo no Autoconceito de Crianças Pré-Escolares.

-escolares são capazes de realizar julgamentos sobre as suas competências em domínios específicos, podendo ser utilizado entre os 4 e os 8 anos de idade. Desta forma, estima a perceção das crianças sobre as suas competências cognitivas e físicas, bem como o grau em que é aceite pela mãe e pelos pares, ao longo de 35 itens. Existe um caderno de imagens para cada um dos sexos, devendo-se igualar o sexo dos desenhos ao sexo da criança que está a responder. $\mathrm{O}$ instrumento foi desenhado de forma a responder a alguns dos desafios metodológicos dos instrumentos para crianças pequenas: por um lado, o seu formato pictórico minimiza a influência das capacidades verbais das crianças, por outro lado, o formato com que as questões são colocadas às crianças reduz os problemas relacionados com a desejabilidade social. O instrumento apresentou propriedades psicométricas aceitáveis (e.g., Harter \& Pike, 1984; Verschueren \& Marcoen, 1999) e foi traduzido e adaptado à população Portuguesa por Mata $\mathrm{e}$ colaboradores (2008). Na nossa investigação, obtiveram-se os seguintes Alfa de Cronbach: Competência Cognitiva $\alpha$ $=0,71$; Competência Física $\alpha=0,73$; Aceitação dos Pares $\alpha=0,79$ e Aceitação Materna $\alpha=0,80$.

\section{Procedimentos de Aplicação da PSPCSA}

Em ambas as idades, o procedimento de coleta dos dados foi semelhante. $\mathrm{O}$ instrumento foi administrado de forma individual, numa sala do estabelecimento de ensino onde era efetuada a recolha de dados, por um investigador do sexo feminino independente das restantes recolhas, bem como das equipas de cotação, e desconhecedor de qualquer informação adicional sobre as crianças. A cada item do instrumento correspondem duas imagens, cujo sexo da personagem principal é igualado ao da criança participante. $\mathrm{O}$ investigador explica à criança o procedimento, mostrando um item de exemplo, com as imagens correspondentes: "Este menino/a é muito bom... MAS este menino/a não é muito bom... Qual deles é mais parecido contigo? E é muito parecido ou mais ou menos parecido contigo?". Desta forma, a criança é levada a optar por um de quatro pontos possíveis de resposta em cada item. Uma vez assegurado que a criança percebeu o procedimento, inicia-se a aplicação dos 35 itens do instrumento.

Cada item do instrumento é cotado numa escala de 4 pontos e os resultados são obtidos em quatro subescalas: (a) Competência Cognitiva; (b) Competência Física; (c) Aceitação dos Pares e (d) Aceitação Materna. Fazendo-se a média das pontuações obtidas nas escalas de competência, obtém-se o compósito de Competência Percebida. Realizando a mesma operação com as pontuações das duas escalas de aceitação obtém-se o compósito de Aceitação Social (Harter \& Pike, 1984). O instrumento foi cotado por um investigador independente das restantes equipas de recolha de dados e de cotação dos instrumentos.

\section{Resultados}

Tendo em conta os objetivos do estudo, as possibilidades fornecidas pelo instrumento utilizado, bem como a reduzida dimensão da amostra, realizaram-se duas séries de análises multivariadas de variância (MANOVA two-way) para medidas repetidas: (a) às quatro subescalas da PSPCSA; (b) aos compósitos de Competência (Cognitiva e Física) e Aceitação (dos Pares e Materna; variáveis dependentes), com o sexo e as idades das crianças como variáveis independentes. Considerou-se um nível de significância $\alpha=0,05$. As classificações da dimensão do efeito e da potência do teste foram feitas, respetivamente, através do $\eta_{P}^{2}$ e do $\pi$, de acordo com Maroco (2010).

\section{Análise Multivariada às Quatro Subescalas da PSPCSA}

As médias e os desvios padrão obtidos em cada subescala, em função da idade e do sexo, estão expostas na Tabela 1. A análise da Tabela 1 permite perceber que os resultados médios obtidos em ambos os sexos e idades, nas quatro subescalas do autoconceito são bastante elevados, encontrando-se acima do ponto médio da escala (2) e bastante próximos do ponto máximo (4).

A análise multivariada apenas detetou resultados marginalmente estatísticos para a idade das crianças, Traço de Pillai $=0,093, F(4,78)=1,999 ; p=0,10 ; \eta^{2}$ $=0,093 ; \pi=0,575$. A análise dos effect size revela que as diferenças devidas à idade são médias e a potência do teste indica-nos que a probabilidade de erro tipo II (probabilidade de não detetar o efeito) é elevada. Desta forma, o valor da potência do teste, aliado à reduzida dimensão da amostra, pode ser responsável pela ausência de significância estatística.

As análises univariadas para os efeitos da idade das crianças no seu autoconceito identificaram a escala de Aceitação Materna como aquela na qual os efeitos da idade são estatisticamente significativos, $F(1,81)=5,697, p=$ $0,019, \eta_{p}^{2}=0,066, \pi=0,655$. A escala de Aceitação dos Pares obteve um resultado marginalmente significativo, $F(1,81)=3,426 ; p=0,068, \eta_{p}^{2}=0,041, \pi=0,448$. Os effect size indicam-nos que estes efeitos são médios e a potência do teste, reduzida, diz-nos que a probabilidade de erro tipo II (probabilidade de não detetar o efeito) é elevada. Em ambas as subescalas os resultados são superiores para as crianças mais novas.

\section{Análise Multivariada aos Compósitos Competência / Aceitação}

A análise multivariada de variância (MANOVA two-way) às quatro subescalas da PSPCSA demonstrou possuir uma potência de teste reduzida. Como tal, era elevada a probabilidade de não detetar um efeito existente. Desta forma, optou-se por repetir a mesma análise aos compósitos Competência e Aceitação.

As médias e os desvios padrão obtidos em cada compósito, em função da idade e do sexo, estão expostas na Tabela 1. Tal como para os dados relativos às quatro subescalas da PSPCSA, os resultados médios obtidos em ambos os sexos e idades, ao nível dos compósitos de competência e aceitação social percebidas são bastante elevados, encontrando-se acima do ponto médio da escala (2) e bastante próximo do ponto máximo (4). 
Tabela 1

Média e Desvio Padrão de cada Subescala do Autoconceito e do Compósito Competência / Aceitação em Função da Idade e do Sexo

\begin{tabular}{cccccc}
\hline & & \multicolumn{2}{c}{4 Anos } & \multicolumn{2}{c}{5 Anos } \\
\cline { 3 - 6 } & & $M$ & $D P$ & $M$ & $D P$ \\
\hline \multirow{2}{*}{ Competência Cognitiva } & Feminino & 3,46 & 0,54 & 3,47 & 0,44 \\
& Masculino & 3,64 & 0,41 & 3,60 & 0,36 \\
Competência Física & Feminino & 3,44 & 0,52 & 3,49 & 0,43 \\
& Masculino & 3,68 & 0,33 & 3,61 & 0,43 \\
Aceitação dos Pares & Feminino & 3,43 & 0,58 & 3,28 & 0,62 \\
& Masculino & 3,65 & 0,47 & 3,57 & 0,41 \\
Aceitação Materna & Feminino & 3,38 & 0,56 & 3,42 & 0,53 \\
& Masculino & 3,57 & 0,50 & 3,42 & 0,58 \\
Competência & Feminino & 3,45 & 0,48 & 3,48 & 0,40 \\
& Masculino & 3,66 & 0,34 & 3,61 & 0,37 \\
Aceitação Social & Feminino & 3,40 & 0,54 & 3,25 & 0,53 \\
& Masculino & 3,61 & 0,44 & 3,50 & 0,47 \\
\hline
\end{tabular}

A análise multivariada detetou resultados estatísticos tanto para a idade das crianças, Traço de Pillai $=0,087$, $F(2,80)=3,799 ; p=0,027 ; \eta_{P}^{2}=0,087 ; \pi=0,676$, como para o seu sexo, Traço de Pillai $=0,073, F(2,80)=3,137$; $p=0,049 ; \eta_{P}^{2}=0,073 ; \pi=0,587$. Em ambos os casos, os effect size são médios e a potência do teste reduzida.

As análises univariadas para os efeitos da idade das crianças no seu autoconceito identificaram a escala de Aceitação como aquela na qual os efeitos da idade são estatisticamente significativos, $F(1,81)=5,515, p=0,021$, $\eta_{p}^{2}=0,064, \pi=0,641$. Os effect size indicam-nos que estes efeitos são médios. A potência do teste é reduzida. As crianças mais novas apresentam resultados mais elevados de Aceitação Social.

Os testes de efeitos between subjects detetaram efeitos do sexo estatisticamente significativos em ambas as escalas: Competência, $F(1,81)=4,957, p=0,029, \eta^{2}=0,058$, $\pi=0,595$, e Aceitação, $F(1,81)=6,073, p=0,016, \eta_{p}{ }_{p}=$ $0,070, \pi=0,683$. A análise dos effect size revela que as diferenças devidas ao sexo em ambas as escalas são médias e superiores para a escala de Aceitação. As potências dos testes são reduzidas. Em ambas as escalas os rapazes tiveram resultados superiores às raparigas.

\section{Discussão}

Um dos aspetos referenciados na literatura, acerca das autoavaliações de crianças pequenas sobre o seu autoconceito, é o facto de estas apresentarem julgamentos irrealisticamente elevados, ainda que normativos (e.g.,
Harter, 2012; Stipek \& Mac Iver, 1989). Os resultados do nosso estudo são concordantes com este facto, com as crianças a apresentarem médias muito elevadas, em todas as subescalas do autoconceito, tanto aos 4 , como aos 5 anos de idade.

Por outro lado, diversos investigadores demonstraram que esta tendência tende a declinar com a idade (e.g., Cole et al., 2001; Eccles et al., 1993; Marsh et al., 1991; Stipek \& Mac Iver, 1989), à medida que as crianças vão sendo capazes de acomodar um maior realismo na forma como se vêem e avaliam. No entanto, a investigação no período pré-escolar sobre este assunto é bastante limitada, e nem sempre se cinge ao mesmo constructo, motivo que pode explicar o facto de a revisão de literatura sobre este assunto encontrar resultados díspares. Os resultados da nossa investigação parecem indicar que esta tendência surge já no fim do período pré-escolar: observou-se uma diminuição estatisticamente significativa no autoconceito das crianças, dos 4 anos para os 5 anos de idade, para as subescalas de aceitação dos pares e aceitação materna e para o compósito de aceitação social percebida. Desta forma, os nossos resultados reforçam os de investigações anteriores (Colwell \& Lindsey, 2003; Mantzicopoulos, 2004). No entanto, é interessante e importante frisar que este declínio não se deu em todos os domínios do autoconceito, mas apenas naqueles que se referem à Aceitação Social. Os domínios da Competência não apresentaram diferenças significativas devidas à idade em nenhuma das análises. Parece-nos que poderão existir três hipóteses explicativas para esse facto. Por um lado, a nossa amostra é bastante reduzida, motivo 
pelo qual a potência de teste é baixa e, logo, a probabilidade de erro tipo II (não detetar um efeito existente) é bastante elevada. Talvez que com uma amostra maior, esse efeito fosse detetado. Por outro lado, diversos autores (e.g., Cole et al., 2001; Marsh et al., 1998) afirmam que o declínio do autoconceito das crianças pode ser diverso, consoante os domínios avaliados, podendo alguns permanecer estáveis. Os nossos resultados poderão corroborar essa hipótese. Finalmente, uma das teorias explicativas para esta diminuição do autoconceito na infância refere-se à acomodação de um maior realismo e à capacidade das crianças se distanciarem dum ideal. Segundo alguns autores, esta diminuição observada na idade escolar deve-se, em parte, à comparação com os resultados e sucesso académicos e à comparação social (Cole et al., 2001; Harter, 2012). Assim, talvez a diminuição dos resultados nos domínios de Competência Percebida (Cognitiva e Física) se observe um pouco mais tarde, apenas com a escolarização. Já no que concerne ao domínio de Aceitação dos Pares, a crescente socialização - e interesse por ela - das crianças, poderá conduzir a que os processos de acomodação do realismo e comparação social operem mais cedo a este nível e, como tal, se observe a diminuição do autoconceito neste domínio. Em relação à Aceitação Materna, hipotetizamos que a diminuição do autoconceito neste domínio se possa dever também, em parte, à crescente sociabilização e interesse por ela, uma vez que alguns dos itens de Aceitação Materna se referem à capacidade da mãe permitir que a criança vá comer, brincar ou dormir em casa de amigos. Por outro lado, com a criança a tornar-se mais velha, mais independente e com a possível chegada de novos irmãos, talvez seja possível que alguns dos cuidados parentais se vão acomodando e alterando e, desta forma, a criança vá percecionando um maior distanciamento materno comparativamente com fases anteriores. Finalmente, o processo de comparação social, com as realidades dos seus pares, que vai conhecendo e percecionando, através da sociabilização, pode permitir que a criança vá alterando a forma como perceciona a sua relação com a sua mãe. Não foi possível testar nenhuma destas hipóteses no nosso estudo.

Por sua vez, e em relação aos efeitos do sexo no autoconceito de crianças pré-escolares, a investigação tem vindo a apontar para a não existência de diferenças (e.g., Ackerman \& Dozier, 2005; Bird \& Reese, 2006; Emídio et al., 2008; Goodvin et al., 2008; Mantzicopoulos, 2004; McCarthy, 1998; Verschueren et al., 1996). No nosso estudo foram detetados efeitos estatisticamente significativos do sexo no autoconceito das crianças, favorecendo os rapazes, na análise dos compósitos de Competência e Aceitação. Estes resultados são contrastantes com os obtidos nas poucas investigações que encontraram diferenças devidas ao sexo no autoconceito de crianças pré-escolares, uma vez que nesses estudos são geralmente as raparigas que reportam valores superiores em todos os domínios ou as diferenças encontradas podem ser explicadas segundo os estereótipos sexuais (e.g., Clark \& Symons, 2000; Gullón-Rivera, 2013; Marsh et al., 2002). Em primeiro lugar, parece-nos que a já referida diminuta dimensão da amostra pode explicar o facto de não se encontrarem diferenças devidas ao sexo na análise às quatro subescalas do autoconceito. Por outro lado, no nosso entender, esse facto prejudica a compreensão dos nossos resultados, já que nos parece que seria fundamental perceber quais os domínios nos quais os rapazes apresentam valores mais elevados. De facto, embora os resultados obtidos nos dois compósitos, Aceitação Social e Competência Percebida, demonstrem que os rapazes apresentam resultados superiores em ambos os domínios, a verdade é que o agregar dos domínios por meio de média aritmética pode enviesar os resultados. Ainda assim, o resultado mais elevado no domínio da Competência Percebida poderá estar de acordo com os estereótipos sexuais, com os rapazes a apresentarem valores mais elevados na competência física e cognitiva.

Resumindo, os nossos dados indicam que, de facto, as crianças pequenas apresentam resultados muito elevados nos diferentes domínios do autoconceito, mas já são capazes de distinguir e apresentar domínios diferenciados, segundo um modelo multidimensional do autoconceito. Por outro lado, os elevados valores do autoconceito parecem começar a declinar já no fim do período pré-escolar, pelo menos nalguns domínios, sugerindo, dessa forma, um desenvolvimento diferenciado do autoconceito, consoante os diferentes domínios avaliados. Finalmente, parecem existir diferenças no autoconceito relacionadas com o sexo das crianças, favorecendo os rapazes, que carecem de um estudo mais aprofundado, de forma a melhor serem compreendidas e explicadas.

No entanto, o nosso estudo apresenta algumas limitações. Por um lado, a dimensão da amostra é pequena para o tipo de análises utilizado. Seria importante e interessante replicar o estudo numa amostra maior para permitir melhor análise e compreensão das diferenças ao nível das quatro subescalas do autoconceito. Por outro lado, por se tratar de uma amostra muito homogénea, toda pertencente a um mesmo estrato sociocultural, não podemos generalizar os nossos resultados. Portanto, seria interessante e importante verificar se estes mesmos resultados seriam replicados numa amostra mais diversificada em termos socioeconómicos. Também a sua replicação em amostras clínicas ou de risco seria importante, para melhor compreender o desenvolvimento do autoconceito em crianças não normativas, a fim de procurar utilizar esse conhecimento no desenvolvimento de programas de intervenção precoce a este nível.

A nossa investigação permitiu alargar a compreensão do desenvolvimento normativo do autoconceito de crianças de idade pré-escolar, ainda que demonstre a necessidade e importância de continuar a realizar este trabalho. $O$ facto de possuirmos dados de cariz longitudinal reforça os resultados obtidos na nossa investigação e parece-nos ser uma vantagem para este tipo de investigações.

\section{Referências}

Ackerman, J., \& Dozier, M. (2005). The influence of foster parent investment on children's representations of self and attachment figures. Applied Developmental Psychology, 26, 507-520. doi:10.1016/j.appdev.2005.06.003 
Bird, A., \& Reese, E. (2006). Emotional reminiscing and the development of an autobiographical self. Developmental Psychology, 42(4), 613-626. doi:10.1037/0012-1649.42.4.613

Byrne, B. (1996). Measuring self-concept across the life span: Issues and instrumentation. Washington, DC: American Psychological Association.

Clark, S., \& Symons, D. (2000). A longitudinal study of Q-sort attachment security and self-processes at age 5 . Infant and Child Development, 9, 91. doi:10.1002/15227219(200006)9:2\%3C91::AID-ICD218\%3E3.3.CO;2-F

Cole, D. A., Maxwell, S. E., Martin, J. M., Peeke, L. G., Seroczynski, A. D., Tram, J. M., \& ...Maschman, T. (2001). The development of multiple domains of child and adolescent selfconcept: A cohort sequential longitudinal design. Child Development, 72(6), 1723-1746. doi:10.1111/1467-8624.00375

Colwell, M., \& Lindsey, E. (2003). Teacher-child interactions and preschool children's perceptions of self and peers. Early Child Development and Care, 173, 249-258. doi:10.1080/03004430303096

Eccles, J., Wigfield, A., Flanagan, C., Miller, C., Reuman, D., \& Yee, D. (1989). Self-concepts, domain values, and selfesteem: Relations and changes at early adolescence. Journal of Personality, 57(2), 283-310. doi:10.1111/j.1467-6494.1989. tb00484.x

Eccles, J., Wigfield, A., Harold, R. D., \& Blumenfeld, P. (1993). Age and gender differences in children's self- and task perceptions during elementary school. Child Development, 64(3), 830-847. doi:10.2307/1131221

Emídio, R., Santos, A. J., Maia, J, Monteiro, V., \& Veríssimo, M. (2008). Auto-conceito e aceitação pelos pares no final do período pré-escolar. Análise Psicológica, 26, 491-499.

Goodvin, R., Meyer, S., Thompson, R. A., \& Hayes, R. (2008). Self-understanding in early childhood: Associations with child attachment security and maternal negative affect. Attachment \& Human Development, 10(4), 433-450. doi:10.1080/14616730802461466

Gullón-Rivera, Á. L. (2013). Puerto Rican kindergartners' selfworth as coded from the Attachment Story Completion Task: Correlated with other self-evaluation measures and ratings of child behavior toward mothers and peers. Attachment \& Human Development, 15(1), 1-23. doi:10.1080/14616734.2 013.743250

Harter, S. (1983). Developmental perspectives on the self-system. In M. Hetherington (Ed.), Handbook of child psychology: Vol. 4. Social and personality development (pp. 275-386). New York: Wiley.

Harter, S. (1985). Manual for the self-perception profile for children. Denver, CO: University of Denver.

Harter, S. (1988). Manual for the self-perception profile for adolescents. Denver, CO: University of Denver.

Harter, S. (2012). The construction of the self. New York: The Guilford Press.

Harter, S., \& Pike, R. (1984). The pictorial scale of perceived competence and social acceptance for young children. Child Development, 55, 1969-1982. doi:10.2307/1129772

Manning, M. (2007). Re-framing how we see student selfconcept. The Education Digest, 72(8), 36-41.

Mantzicopoulos, P. (2004). I am really good at puzzles, but I don't get asked to play with others: Age, gender, and ethnic differences in head start children's self-perceptions of competence. Journal of Genetic Psychology, 165(1), 51-65. doi:10.3200/ GNTP.165.1.51-66

Maroco, J. P. (2010). Análise estatística com o PASW Statistics (ex-SPSS). Pêro Pinheiro, Portugal: ReportNumber.
Marsh, H., \& Hattie, J. (1996). Theoretical perspectives on the structure of self-concept. In B. Bracken (Ed.), Handbook of self-concept: Developmental, social, and clinical considerations (pp. 38-90). New York: Wiley.

Marsh, H. W., Craven, R. G., \& Debus, R. (1991). Self-concepts of young children 5 to 8 years of age: Measurement and multidimensional structure. Journal of Educational Psychology, 83(3), 377-392. doi:10.1037/0022-0663.83.3.377

Marsh, H. W., Craven, R., \& Debus, R. (1998). Structure, stability, and development of young children's self-concepts: A multicohort-multioccasion study. Child Development, 69(4), 1030-1053. doi:10.2307/1132361

Marsh, H. W., Ellis, L., \& Craven, R. (2002). How do preschool children feel about themselves? Unraveling measurement and multidimensional self-concept structure. Developmental Psychology, 38(3), 376-393. doi:10.1037/0012-1649.38.3.376

Marsh, H. W., Smith, I. D., \& Barnes, J. (1985). Multidimensional self-concepts: Relations with sex and academic achievement. Journal of Educational Psychology, 77(5), 581-596. doi:10.1037/0022-0663.77.5.581

Mata, L., Monteiro, V., \& Peixoto, F. (2008). Escala de auto-conceito para crianças em idade pré-escolar e $1 .^{\circ} / 2 .^{\circ}$ ano de escolaridade de Harter \& Pike (1981, 1984). In A. P. Machado, C. Machado, L. S. Almeida, M. Gonçalves, S. Martins, \& V. Ramalho (Eds.), Actas da XIII Conferência Internacional Avaliação Psicológica: Formas e Contextos. Braga, Portugal: Associação dos Psicólogos Portugueses.

McCarthy, G. (1998). Attachment representations and representations of the self in relation to others: A study of preschool children in inner-city London. British Journal of Medical Psychology, 71(1), 57-72. doi:10.1111/j.2044-8341.1998. tb01367.x

Oyserman, D., Elmore, K., \& Smith, G. (2012). Self, self-concept, and identity. In M. R. Leary \& J. P. Tagney, (Eds.), Handbook of self and identity (pp. 69-104). New York: Guilford Press.

Pinto, A., Gatinho, A., Silva, F., Veríssimo, M., \& Santos, A. J. (2013). Vinculação e modelo interno dinâmico do self em crianças de idade pré-escolar. Psicologia, Saúde \& Doença, 14, 515-528.

Peixoto, F. (2003). Auto-estima, autoconceito e dinâmicas relacionais em contexto escolar (Tese de doutorado em Psicologia, Universidade do Minho, Braga, Portugal).

Stipek, D., \& Mac Iver, D. (1989). Developmental change in children's assessment of intellectual competence. Child Development, 60(3), 521. doi:10.2307/1130719

Verschueren, K., \& Marcoen, A. (1999). Representation of self and socioemotional competence in kindergartners: Differential and combined effects of attachment to mother and to father. Child Development, 70, 183-201. doi:10.1111/14678624.00014

Verschueren, K., Marcoen, A., \& Schoefs, V. (1996). The internal working model of the self, attachment, and competence in five-year-olds. Child Development, 67(5), 2493-2511. doi: $10.2307 / 1131636$ 